INPLASY

PROTOCOL

To cite: Ávila Carvalho et al. Effects of jump training on the performance of dancers and gymnasts: A systematic review. Inplasy protocol 202140141. doi:

10.37766/inplasy2021.4.0141

Received: 29 April 2021

Published: 29 April 2021

Corresponding author:

Lurdes Ávila Carvalho

lurdesavila2@gmail.com

Author Affiliation:

University of Coimbra, Faculty of Sport Science and Physical Education

Support: None.

Review Stage at time of this submission: Data analysis.

Conflicts of interest:

None declared.

\section{EFFECTS OF JUMP TRAINING ON THE PERFORMANCE OF DANCERS AND GYMNASTS: A SYSTEMATIC REVIEW}

Ávila Carvalho, L1; Sá, L2; Sarmento, H3; Rama, L4.

Review question / Objective: To examine the effects of Jump training programs on dancers and gymnasts.

Condition being studied: Besides the dramatic and artistic performance of dancers, dance demands high levels of high jumping capacity. The high-performance level in ballet and dance is also pointed out as one of the predictors of performance. Those who are skilled to jump higher can increase the number and complexity of motor skills. They can perform and improve the perception of the aesthetic and artistic component of the choreography. The jump ability is associated with the dancer performance level. In Gymnasts, an increased jumping performance can be critical for competition purposes since this sport requires explosive strength expressed in the vertical jump. For both activities, Dance and Gymnastics, the importance of specificity of the jump training protocols is essential to improve the jump height and guarantee that they have a transfer to the specificity of the activities.

INPLASY registration number: This protocol was registered with the International Platform of Registered Systematic Review and Meta-Analysis Protocols (INPLASY) on 29 April 2021 and was last updated on 29 April 2021 (registration number INPLASY202140141).

\section{INTRODUCTION}

Review question / Objective: To examine the effects of Jump training programs on dancers and gymnasts.

Rationale: Increased scientific awareness of the relevance of jump training, the lack of systematic reviews studies targeted to dance, ballet and gymnastics and the lack of comprehensive analyses of their jump performance and the effects of jump specific training, including the transfer to specific needs of these activities.

Condition being studied: Besides the dramatic and artistic performance of dancers, dance demands high levels of 
high jumping capacity. The highperformance level in ballet and dance is also pointed out as one of the predictors of performance. Those who are skilled to jump higher can increase the number and complexity of motor skills. They can perform and improve the perception of the aesthetic and artistic component of the choreography. The jump ability is associated with the dancer performance level. In Gymnasts, an increased jumping performance can be critical for competition purposes since this sport requires explosive strength expressed in the vertical jump. For both activities, Dance and Gymnastics, the importance of specificity of the jump training protocols is essential to improve the jump height and guarantee that they have a transfer to the specificity of the activities.

\section{METHODS}

Search strategy: Database searches were conducted for relevant publication prior to February 22, 2021, in the following databases: Web of Science, SCOPUS and SPORTDiscus. The search strategy include the following keywords: (gymnast* OR danc* OR Ballet) AND ("force-velocity" OR "force velocity" OR jump OR explosive OR power). Duplicates were removed, and the search results were analysed according to the eligibility criteria. In selecting studies for inclusion, a review of all relevant titles was conducted before examining the abstracts and then the full texts. Only peerreviewed articles were included in the systematic review. Besides, we included only the most recent researches between 2010 and 2021. An external expert was contacted to verify the final list of references included in this systematic review and indicate any study that was not detected through our search.

Participant or population: Ballet and dance performers and gymnasts from all gymnastics disciplines from any age or sex, without injury, illness or other clinical condition.

Intervention: All training programs were included, with no limit for training sessions.
No restriction to frequency (number of sessions per week) or intervention duration, as well as no limitation to the type of jump training programs presented in the articles found. Also, we added crossreferenced papers that characterise the dance and gymnastics jump capacity.

Comparator: Subjects were compared with controls.

Study designs to be included: Randomized controlled trials.

Eligibility criteria: To be eligible for inclusion in this systematic review, studies need to meet the following criteria : 1) contain relevant information to the topic; 2) developed with athletes from gymnastics, or performers from ballet or dance from any age or sex, without injury, illness or other clinical condition; 3) Include jumping assessment; 4) were peer reviewed, original, full-text studies written in English or Spanish Languages. The articles were excluded if: 1) were developed in population in the fields not related with gymnastic or dance and ballet; 2) evaluated other physical abilities than jumping, 3) were written in other languages than English or Spanish, 4) Reviews, letters to editors, trial registrations, proposals for protocols, editorials, book chapters or conference abstracts. In case of divergence between the authors, a discussion was held regarding the inclusion of articles until a consensus was reached.

Information sources: Database searches were conducted using Web of Science, SCOPUS and SPORTDiscus electronic databases for relevant publications.

Main outcome(s): The height of the jump was chosen as the primary outcome.

Additional outcome(s): The absence of effects after the training program was also extracted, in case of intervention studies or if reported. Assessment instruments, duration and content of the training protocol data were also considered, as 
they were related to the jump measure and the studies results.

Data management: All data were exported to reference manager software (EndNoteTM X8, Clarivate Analytics, Philadelphia, PA, USA). Any duplicates were eliminated either automatically or manually. We also shared spreadsheet with the whole team.

Quality assessment / Risk of bias analysis: To assess the studies quality, we used the quality form and risk of bias proposed by Sarmento et al. (2018), adapted from the original version developed by Law et al. (1998). The articles were assessed based on purpose (item 1), the relevance of background literature (item 2), appropriateness of study design (item 3), the sample studied (items 4 and 5), use of informed consent procedure (item 6), outcome measures (item 7 and 8), method description (item 9), the significance of results (item 10), analysis (item 11), practical importance (item 12), report of dropouts (item 13), conclusions (item 14), practical implications (item 15), and limitations (item 16). All 16 quality criteria were scored on a binary scale $(0 / 1)$, wherein two of those criteria (items 6 and 13) presented the option: 'If not applicable, assume 3'. All articles were classified as (1) low methodological quality, with a score $\leq 50 \%$; (2) good methodological quality, with a score of between 51 and $75 \%$; and (3) excellent methodological quality, with a score $>75 \%$. First, one researcher extracted the data from included studies and a second and third researchers then checked the extracted data. Disagreements were resolved by consensus.

Strategy of data synthesis: The data was divided into activities specificity (this means if the data were from ballet performers, dancers or gymnasts) and according to the training intervention duration.

Subgroup analysis: The data were also divided according to the type of training applied and instruments used to evaluate the jump height.

Sensitivity analysis: No analysis to report.

Language: Only articles published in the English and Spanish languages were considered.

Country(ies) involved: Portugal.

Keywords: Jump height, Power, Explosive strength, Ballet, Dance, Gymnastics.

Contributions of each author:

Author 1 - Lurdes Ávila Carvalho - lead the project, made the data search, the methodological analysis and the qualitative criteria, provided feedback and wrote the manuscript.

Email: lurdesavila2@gmail.com

Author 2 - Luciana Sá - drafted the tables, helped in the qualitative criteria.

Email: luciana.fmelosa@gmail.com

Author 3 - Hugo Sarmento - The author provided feedback and approved the final manuscript.

Email: hg.sarmento@gmail.com

Author 4 - Luís Rama - The author did the data search, helped in the qualitative criteria, provided feedback and approved the final manuscript.

Email: luisrama@fcdef.uc.pt 